\title{
A Low-cost and Portable System for 3D Reconstruction of Texture-less Objects
}

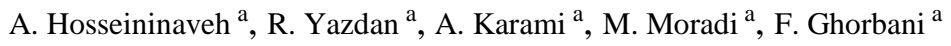 \\ aK.N.Toosi University of Technology, Geodesy \&Geomatics Faculty, Tehran, Iran ali_hosseini_naveh@yahoo.com - \\ ro.yazdan@gmail.com - ali.karami.surveying@gmail.commm136988@yahoo.com - f_ghorbani95@yahoo.com
}

KEY WORDS: 3D modelling, texture-less objects, low-cost system, turntable, stepper motor, Structure from Motion

\begin{abstract}
The optical methods for 3D modelling of objects can be classified into two categories including image-based and range-based methods. Structure from Motion is one of the image-based methods implemented in commercial software. In this paper, a low-cost and portable system for 3D modelling of texture-less objects is proposed. This system includes a rotating table designed and developed by using a stepper motor and a very light rotation plate. The system also has eight laser light sources with very dense and strong beams which provide a relatively appropriate pattern on texture-less objects. In this system, regarding to the step of stepper motor, images are semi automatically taken by a camera. The images can be used in structure from motion procedures implemented in Agisoft software. To evaluate the performance of the system, two dark objects were used. The point clouds of these objects were obtained by spraying a light powders on the objects and exploiting a GOM laser scanner. Then these objects were placed on the proposed turntable. Several convergent images were taken from each object while the laser light sources were projecting the pattern on the objects. Afterward, the images were imported in VisualSFM as a fully automatic software package for generating an accurate and complete point cloud. Finally, the obtained point clouds were compared to the point clouds generated by the GOM laser scanner. The results showed the ability of the proposed system to produce a complete 3D model from texture-less objects.
\end{abstract}

\section{Introduction}

At the recent years, 3D Modeling techniques and systems have been rapidly improved in diverse applicationssuch as in medicine for accurate $3 \mathrm{D}$ reconstruction of body organs and investigating muscle tissue changes for medical diagnosis(Sansoni, Trebeschi, \& Docchio, 2009);(Chang, Detchev, \& Habib, 2008); (Barazzetti, Gianinetto, \& Scaioni, 2012; Chang et al., 2008; Sansoni et al., 2009), in cultural heritage for preserving and repairing cultural heritage buildings(Arias, Ordóñez, Lorenzo, Herraez, \& Armesto, 2007) and producing the digital archive of museum objects for documentation, visualization and virtual museums (Chang et al., 2008); (Barazzetti et al., 2012); in industry for reverse engineering of industrial components, criminal investigation(Sansoni et al., 2009), fashion and clothing(D’Apuzzo \& Gruen, 2009);(Gu et al., 1998), and other fields such as geology and entertainment industry (Chadwick, Haumann, \& Parent, 1989);(Volino, Thalmann, Jianhua, \& Thalmann, 1998);(Sansoni et al., 2009); (Remondino, 2011).

3D Modeling can be classified into two categories of image-based and range-based techniques(Remondino \& El-Hakim, 2006). Image-based techniques can generate an accurate 3D model which 3D coordinates of objects are determined by a set of 2D overlapped images from different views. Structure from Motion (SfM) is one of the most common techniques in this category that has been implemented in different software. This technique and Dense Multi-View Stereo (DMVS) techniques can deliver a very accurate and dense point cloud from the object (Ali Hosseininaveh Ahmadabadian, Robson, Boehm, Shortis, et al., 2013).
When the surface of the interesting object does not have a recognizable texture, finding corresponding feature points in images as one of the crucial steps in SfM techniques, faces difficulties (Brown, Burschka, \& Hager, 2003).It can mention that if the object is texture-less, all of feature point algorithms such as SIFT will face difficulties to find key feature points(Lowe, 2004). In order to solve this problem, different methods have been proposed such as structure light or pattern protection. In these methods, projecting a predefined pattern on an object and comparing the reflected pattern from the object with the pre-defined pattern can be used to extract depth information(Le Moigne \& Waxman, 1985); (Morita, Yajima, \& Sakata, 1988); (Maruyama \& Abe, 1993); (Boyer \& Kak, 1987); (Tang \& Hung, 1990); (Srinivasan, Liu, \& Halioua, 1984). Similar technique can be used in SfM techniques by projecting a very dense and texture-full pattern on the object before capturing the images.

Although the most of SfM software is easy to use for nonprofessionals, capturing suitable images for this software make the process time consuming and difficult for the users. This issue can be very important where a large number of small complex objects such as museum objects are needed to be modeled.

This paper aims to introduce a new, simple and applicable system for 3D reconstruction of small texture-less objects. To emphasis the advantages of the proposed system in comparison with available systems, a literature review is provided in the next section. Then, the proposed system is described and evaluated in the rest of this article. 


\section{Background}

Systems for 3D reconstruction can be classified into three classes including the systems working with active sensor placement(Mahadevan, Pandzo, Bennamoun, \& Williams, 2001);(Scott, Roth, \& Rivest, 2003);(Chan \& Samaan, 2004);(Zexiao, Jianguo, \& Qiumei, 2005);(Paul, Webb, Liu, \& Dissanayake, 2011) and (Karaszewski, Sitnik, \& Bunsch, 2012), the system working with passive sensors(Zhuang, Wang, \& Roth, 1995);(Chen \& Li, 2004), and the systems working with both active and passive sensor(Larsson \& Kjellander, 2008);(Kasper, Xue, \& Dillmann, 2012).Generally, range-based systems are more expensive than the image-based systems which use ordinary cameras for producing 3D model(Paulus, Behmann, Mahlein, Plümer, \& Kuhlmann, 2014).

At optoelectronics laboratory in Brescia university, a system was designed consisting of a single optical head with a camera and a projector for quality control that has exploited a triangulation geometry, gray code, phase shifting patterns and a rotation table to generate a 3D model from an interesting object (Sansoni \& Patrioli, 2000);(Krattenthaler, Mayer, \& Duwe, 1994). In order to monitor the deformity of human spine for scoliosis diagnosis, a system including four metal arms which had seven low-cost cameras and a projector for each was designed(Chang et al., 2008). The projectors projected an artificial pattern on the patient's body and all the cameras captured the images simultaneously. Although the system was very useful in comparison with the X-ray scanning, the system is expensive due to using 16 cameras and 4 projectors.

An integration of a $3 \mathrm{D}$ vision sensor and Coordinate Measuring Machine(CMM) was performed to provide a 3D reconstruction system for inverse engineering of free form and complex surfaces(Carbone, Carocci, Savio, Sansoni, \& De Chiffre, 2001). In this system, generating the final CAD model was accomplished using integration $\mathrm{CMM}$ and $3 \mathrm{D}$ vision technique including a video camera, LCD projector and rotation stage to acquire a point cloud with accuracy of $0.1 \mathrm{~mm}$ and resolution of $70 \mu \mathrm{m}$. Afterward, this point cloud was preprocessed to remove noises. Although the system can provide the 3D CAD model from the object with minimum of human intervention, the system is expensive and non-portable due to using CMM.

One of the most recent structured light systems that can deliver a complete 3D model from planets was designed in California University(Nguyen, Slaughter, Max, Maloof, $\&$ Sinha, 2015).Planet3D modeling was achieved in this system by using structure light technique with exploiting five pairs of camera mounted on an arc holding. In this system, an optical projector with random dot pattern fed by an electrical adaptor was used. Plants placed on a turntable that can rotate 360 degrees. This system was able to generate a complete 3D planet model by taking stereo images. In this system, in addition of using a lot of cameras, plenty of space is occupied and portable functionality is problematic.

One of the automatic designed robots which worked at cultural heritages field was designed by Karaszewski et al. This robot has six degree of freedom and one rotation table. NBV algorithm was used in this robot for positioning measurement scanners at a certain location around the object used. This system is collision-free and is expensive (Karaszewski et al., 2012); (graphics., 2015). Stanford Spherical Gantry is another robot with four degree of freedom and two arms that can be controlled by computer and was placed on rotation table. Designing of this system let camera to cover entire surface of sphere (except 10 degree of bottom part) to the center of rotation table and can capture images from small object for modeling(Levoy, 2002). Hosseininaveh (2014) designed INDRo robot with six degree of freedom to produce accurate and complete point clouds from small objects with complex surface. The robot included a package called IND to design an imaging network. Images could be captured at designed positions using two industrial cameras, a pan-tilt unit, a goniometer, a turn table and two linear stages, (Hosseininaveh et al., 2014). Although the robot could automatically deliver a complete 3D model from small objects, it was not successful in modeling of texture-less objects and also this system was not portable.

In this paper, we developed a low-cost and portable semiautomatic imaging system for point cloud generation of small and texture-less objects by using a stepper motor and turntable which the object is placed on and under the specified certain angles of rotation. Using of Stepper motor and the turntable at this system get work convenience, less operator intervention and reduce time of the modeling process. In order to overcome the matching problem of texture-less object in the process of modeling, projection of the appropriate pattern on the object is used.

\section{Proposed system}

The proposed system is a low-cost and portable for 3D modeling of small and texture-less object. As it is shown in figure1, the system consists of the following components: a stepper motor, a circular plate, dot lasers with simple and dense patterns, scale bar, a digital camera and computer.

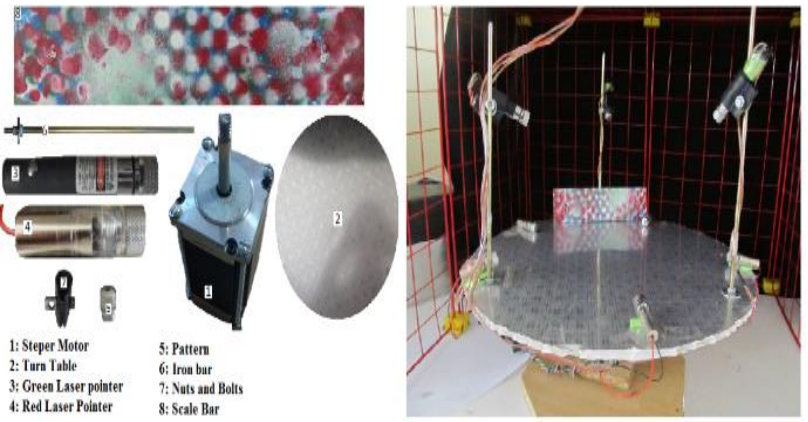

Figure1- general components of system

Stepper motor has been used in this system is a hybrid that has the capability of resisting $4 \mathrm{~kg}$ weight and can rotate in clockwise and counter clockwise.

A hybrid engine stepper motor was exploited in this system with carrying capacity(payload)of $4 \mathrm{~kg}$ and can rotate in both 
clockwise and counter clockwise. An UPVC circular plate with $60 \mathrm{~cm}$ radius was installed on this stepper motor. For the installation of the circular plate, the main axis of stepper motor got wiredrawn and connected to circular plate with nuts and bolts. Two kinds of dot lasers(red and green) were used to solve matching problems of texture-less objects. Five red dot lasers with simple patterns were placed over and near to the edge of the circular plate to project their patterns on the surface of the object from the horizon. Three green dot lasers were placed on top of three iron bars with a height of $30 \mathrm{~cm}$ mounted at the edge of the circular plate to project their simple patterns from tilted angles in respect to the object. The power supplies of laser patterns have been changed from batteries to adaptors to make sure the luminance of the patterns is stable during capturing images. Also, a texture-full scale bar with a length of $149.09 \mathrm{~cm}$ was used to resolve the scale issue in the Structure from Motion process.

The general process of proposed system is shown in figure 2 and figure 3.The command sent to the driver of the stepper motor from a workstation computer prompts the stepper motor to rotate by a specific angle defined by the user. Then, while the objects placed on the center of the rotation table are fully covered with patterns projected by dot lasers, an image is captured by a digital camera controlled by the workstation computer. This operation continues until the turn table complete as whole round (400 grades).Afterward, by changing the elevation of the camera, the imaging process is repeated for the second ring. In order to have a better reflection of the patterns on the object, the turn table is placed under a frame with a black cover. Having captured the images, 3D reconstruction of the object can be completed by transferring the images to SfM software (e.g. VisualSFM or Agisoft). The most essential issue to have a very complete and accurate model is imaging process which we discuss below.

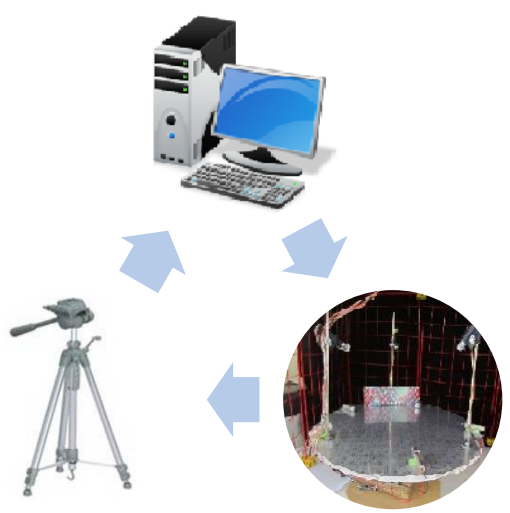

Figure 2 - general processes of proposed system

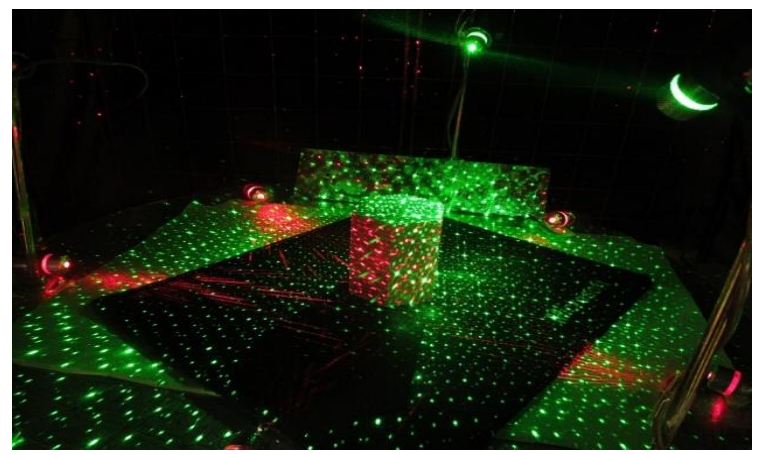

Figure3- The dot patterns projected on the object

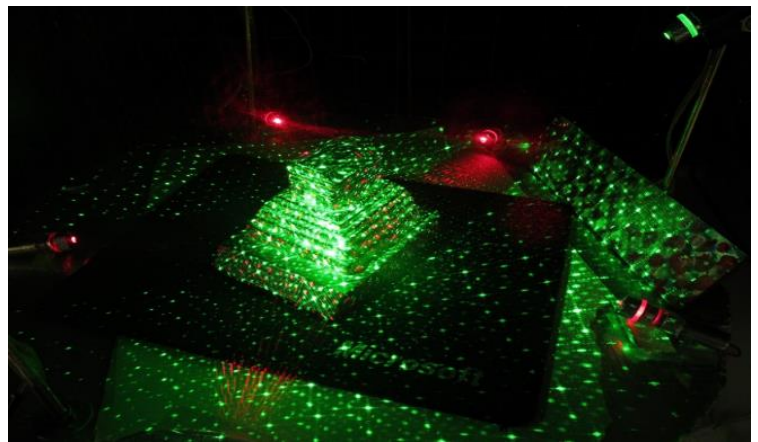

Figure 4-The dot patterns projected on the object

\section{3-1 Imaging Network Design for the system}

In order to have an accurate and complete $3 \mathrm{D}$ model, in addition to have an appropriate digital camera, it is essential to configure the most suitable places for the camera. In close range photogrammetry this issue is known as imaging network design. Fraser (1984) has expressed non-linearity and multimodality problems in photogrammetric network design (PND) and to solve these problems, he proposed certain constraints for an ideal imaging network for 3D measurements of certain points. These constraints were divided into three categories consist of range-related (image scale, resolution, camera Field of View, Depth of Field, number and distribution of points and workspace), visibility-related (incidence angle, occluded area and camera FOV) and accessibility-related (physical constraints of space, obstructions) constraints (Saadatseresht, Fraser, Samadzadegan, \& Azizi, 2004).An ideal imaging network for generating an accurate and dense point clouds from images has also been discussed by previous researchers (Ali Hosseininaveh Ahmadabadian, Robson, Boehm, \& Shortis, 2014)who proposed a system called Imaging Network Designer (IND) based on the mentioned constraints.IND can both design a complete imaging network and cluster and select vantage images from a set of available images(Ali Hosseininaveh Ahmadabadian, Robson, Boehm, \& Shortis, 2013); (Ali Hosseininaveh Ahmadabadian et al., 2014).IND can both compute the optimum range from the objects for each camera and suggest the vantage positions and orientations for the camera. In the proposed system, IND can be used to find the optimum range from the objects. Moreover, because the camera is fixed on a tripod in front of the turntable, there is no need to design a complex geometrical imaging configuration for the 
system. An effective strategy could be to capture images in two rings by changing the height of the camera. The pattern projected on the object must have an adequate distribution and dispersion. F-stop and shutter speed are two significant parameters to obtain high quality images from the object covered with this pattern. The optimal F-stop and shutter speed, estimated in a try and error procedure, were set 3.4 and 0.3 seconds respectively.

In addition to the network design, the speed of stepper motor and its rotation angle are two other effective parameters. Increasing rotation angle will lead to decease in the number of images and processing time. However, if this value is bigger than 30 degrees, the reconstruction process will face errors because the maximum suitable angles for viewing angles between two camera positions for SIFT should be less than 30 degrees(Morel \& Yu, 2009).On the other hand, if the speed of rotation is set higher than a specific value, the stability of the system will be in trouble. The optimum values for these parameters were estimated based on a trial and error method. The suitable speed and rotation angle were obtained 60 Round per Minuets (RPM) and 20 grades respectively.

Last but not least, in 3D reconstruction of Symmetrical objects when the object rotates on the turntable, the similar pattern and similar shape will be repeated in different images. This issue results in failures in computing exterior orientation parameters for some of the images. To solve this problem, a scale bar is made from an iron bar and painted in different colors and placed beside the objects. This scale bar can be used to resolve the scale issue in SfM procedure as well.

\section{Experiments and Results}

To evaluate the performance of the system, two texture-less objects (a hexagon metallic object and stone statue)were considered to be reconstructed. The objects were scanned with an accurate commercial laser scanner known as GOM ATOS Compact Scan 2M. The point spacing of the scanner is between 0.021 and $0.615 \mathrm{~mm}$ and accurate enough to provide a ground truth dataset for this test.

A digital camera, Canon Powershot SX130, with 12 megapixels images were used to capture images from the objects. The images were captured in two rings in an optimum range distance from the objects while the patterns were projected on the object. As it was mentioned the rotation angle was set 20 grades and for a whole round (400 grades) 20 images were captured in each rings. The 40 captured images were then imported in Photoscan Agisoft software to provide a 3D model from the objects. The point cloud generated by this software was then compared with the point cloud achieved by GOM laser scanner. The results of this comparison are shown in Figure 5.

To compare the generated point cloud by proposed system and the ground truth data provided with the GOM laser scanner, GOM inspect software was used. A plane was fitted to each face of hexagon metallic object and the deviations between the plane and the points on surface were computed and compared with the deviations of corresponding sides on the ground truth data of hexagon and a plane. Two profiles in the middle of the stone statue are used to evaluate the models obtained for this object from both GOM scanner and the proposed system. The standard deviation for hexagon metallic object stone statue were $100 \mu \mathrm{m}$ and150 $\mu \mathrm{m}$ respectively. One of advantages of the proposed system is that it can be used for a wide range of cameras with a diversity of resolutions.

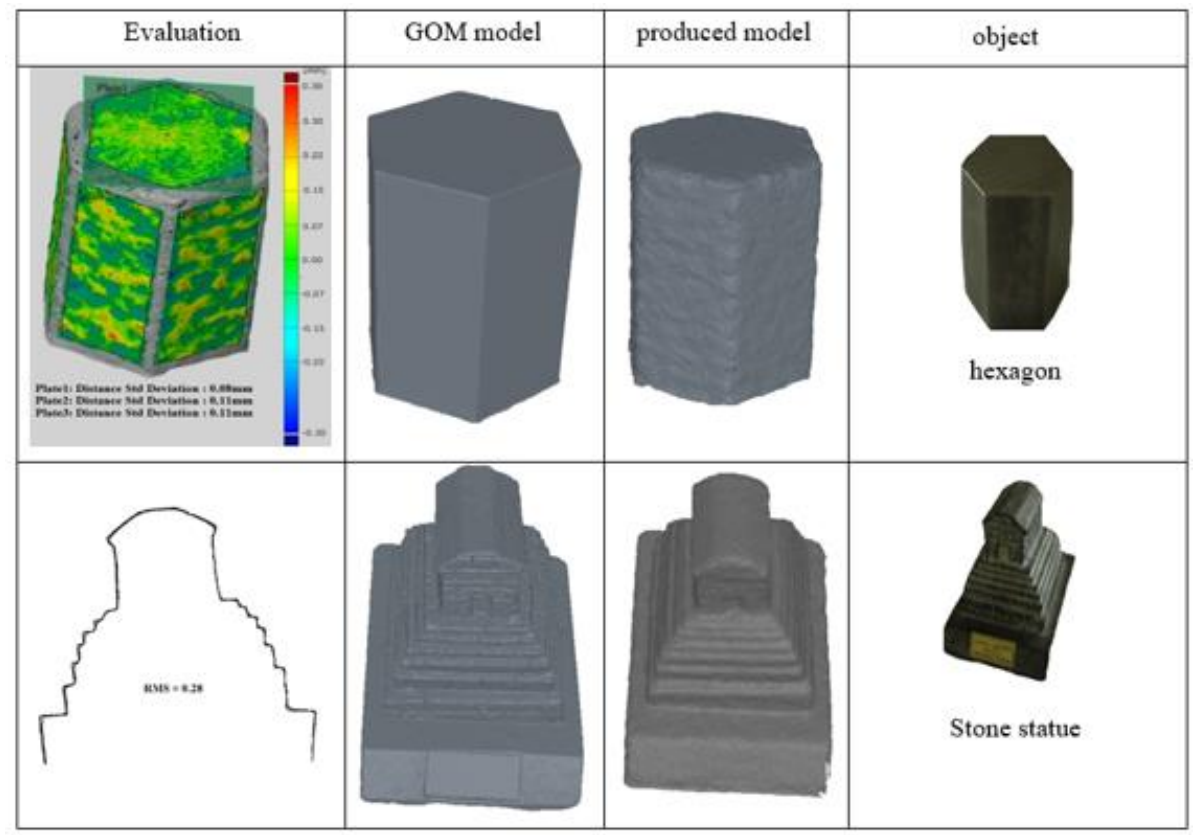

Figure 5-Comparing the point clouds generated with the proposed system and the GOM laser scanner 


\section{Conclusion}

The purpose of this paper is to provide a portable and affordable system for 3D reconstruction of small and textureless objects. The main contribution of this research is to exploit low cost instruments such as dot lasers with pattern to overcome the problem of texture-less objects for 3D reconstruction. Moreover, the cost, portability, automation and accuracy of the proposed system make it outstanding from similar recent systems in this field. The use of visible patterns has provided this opportunity to use any imaging sensor with a diversity of resolutions. Therefore, if a digital camera with a better configuration is used in the proposed system, better results than $150 \mu \mathrm{m}$ can be achieved.

\section{6- Reference}

Arias, P., Ordóñez, C., Lorenzo, H., Herraez, J., \& Armesto, J. (2007). Low-cost documentation of traditional agroindustrial buildings by close-range photogrammetry. Building and environment, 42(4), 1817-1827.

Barazzetti, L., Gianinetto, M., \& Scaioni, M. (2012). Automatic image-based 3D modeling for medical applications. Paper presented at the Biomedical Engineering and Informatics (BMEI), 2012 5th International Conference on.

Boyer, K. L., \& Kak, A. C. (1987). Color-encoded structured light for rapid active ranging. Pattern Analysis and Machine Intelligence, IEEE Transactions on(1), 1428.

Brown, M. Z., Burschka, D., \& Hager, G. D. (2003). Advances in computational stereo. Pattern Analysis and Machine Intelligence, IEEE Transactions on, 25(8), 993-1008.

Carbone, V., Carocci, M., Savio, E., Sansoni, G., \& De Chiffre, L. (2001). Combination of a vision system and a coordinate measuring machine for the reverse engineering of freeform surfaces. The International Journal of Advanced Manufacturing Technology, 17(4), 263-271.

Chadwick, J. E., Haumann, D. R., \& Parent, R. E. (1989). Layered construction for deformable animated characters. Paper presented at the ACM Siggraph Computer Graphics.

Chan, V. H., \& Samaan, M. (2004). Spherical/cylindrical laser scanner for geometric reverse engineering. Paper presented at the Electronic Imaging 2004.

Chang, Y.-C., Detchev, I., \& Habib, A. (2008). A Photogrammetric System for 3D Reconstruction of a Scoliotic Torso. Paper presented at the Proceedings of the ASPRS 2009 Annual Conference.

Chen, S., \& Li, Y. (2004). Automatic sensor placement for model-based robot vision. Systems, Man, and Cybernetics, Part B: Cybernetics, IEEE Transactions on, 34(1), 393-408.

D'Apuzzo, N., \& Gruen, A. (2009). Recent advances in 3D full body scanning with applications to fashion and apparel. Optical 3-D Measurement Techniques IX.

Gu, J., Chang, T., Mak, I., Gopalsamy, S., Shen, H. C., \& Yuen, M. M.-F. (1998). A 3D reconstruction system for human body modeling Modelling and Motion Capture Techniques for Virtual Environments (pp. 229-241): Springer.
Hosseininaveh, A., Sargeant, B., Erfani, T., Robson, S., Shortis, M., Hess, M., \& Boehm, J. (2014). Towards fully automatic reliable 3D acquisition: From designing imaging network to a complete and accurate point cloud. Robotics and Autonomous Systems, 62(8), 1197-1207.

Hosseininaveh Ahmadabadian, A., Robson, S., Boehm, J., \& Shortis, M. (2013). Image selection in photogrammetric multi-view stereo methods for metric and complete $3 D$ reconstruction.

Hosseininaveh Ahmadabadian, A., Robson, S., Boehm, J., \& Shortis, M. (2014). Stereo-imaging network design for precise and dense $3 \mathrm{~d}$ reconstruction. The Photogrammetric Record, 29(147), 317-336. doi: 10.1111/phor.12076

Hosseininaveh Ahmadabadian, A., Robson, S., Boehm, J., Shortis, M., Wenzel, K., \& Fritsch, D. (2013). A comparison of dense matching algorithms for scaled surface reconstruction using stereo camera rigs. ISPRS Journal of Photogrammetry and Remote Sensing, 78(0), 157-167. doi: http://dx.doi.org/10.1016/j.isprsjprs.2013.01.015

Karaszewski, M., Sitnik, R., \& Bunsch, E. (2012). On-line, collision-free positioning of a scanner during fully automated three-dimensional measurement of cultural heritage objects. Robotics and Autonomous Systems, 60(9), 1205-1219.

Kasper, A., Xue, Z., \& Dillmann, R. (2012). Semi automatic object modeling for a service robot Towards Service Robots for Everyday Environments (pp. 167-179): Springer.

Krattenthaler, W., Mayer, K., \& Duwe, H. (1994). 3D-surface measurement with coded light approach. Paper presented at the Proceedings of the 17th meeting of the Austrian Association for pattern recognition on Image analysis and synthesis.

Larsson, S., \& Kjellander, J. A. (2008). Path planning for laser scanning with an industrial robot. Robotics and Autonomous Systems, 56(7), 615-624.

Le Moigne, J., \& Waxman, A. M. (1985). Multi-resolution grid patterns for building range maps: Society of Manufacturing Engineers.

Levoy, M. (2002). The stanford spherical gantry.

Lowe, D. (2004). Distinctive Image Features from ScaleInvariant Keypoints. International Journal of Computer Vision, 60(2), 91-110. doi: 10.1023/B:VISI.0000029664.99615.94

Mahadevan, S., Pandzo, H., Bennamoun, M., \& Williams, J. A. (2001). A 3D acquisition and modelling system. Paper presented at the Acoustics, Speech, and Signal Processing, 2001. Proceedings.(ICASSP'01). 2001 IEEE International Conference on.

Maruyama, M., \& Abe, S. (1993). Range sensing by projecting multiple slits with random cuts. Pattern Analysis and Machine Intelligence, IEEE Transactions on, 15(6), 647-651.

Morel, J.-M., \& Yu, G. (2009). ASIFT: A New Framework for Fully Affine Invariant Image Comparison. SIAM Journal on Imaging Sciences, 2(2), 438-469. doi: doi:10.1137/080732730

Morita, H., Yajima, K., \& Sakata, S. (1988). Reconstruction of surfaces of 3-d objects by m-array pattern projection 
method. Paper presented at the Computer Vision., Second International Conference on.

Nguyen, T. T., Slaughter, D. C., Max, N., Maloof, J. N., \& Sinha, N. (2015). Structured Light-Based 3D Reconstruction System for Plants. Sensors, 15(8), 18587-18612.

Paul, G., Webb, S., Liu, D., \& Dissanayake, G. (2011). Autonomous robot manipulator-based exploration and mapping system for bridge maintenance. Robotics and Autonomous Systems, 59(7), 543-554.

Paulus, S., Behmann, J., Mahlein, A.-K., Plümer, L., \& Kuhlmann, H. (2014). Low-cost 3D systems: suitable tools for plant phenotyping. Sensors, 14(2), 30013018.

Remondino, F. (2011). Heritage recording and 3D modeling with photogrammetry and 3D scanning. Remote Sensing, 3(6), 1104-1138.

Remondino, F., \& El-Hakim, S. (2006). Image-based 3D Modelling: A Review. The Photogrammetric Record, 21(115), 269-291.

Saadatseresht, M., Fraser, C. S., Samadzadegan, F., \& Azizi, A. (2004). Visibility Analysis In Vision Metrology Network Design. The Photogrammetric Record, 19(107), 219-236. doi: 10.1111/j.0031868X.2004.00280.x

Sansoni, G., \& Patrioli, A. (2000). Noncontact $3 d$ sensing of free-form complex surfaces. Paper presented at the Photonics West 2001-Electronic Imaging.

Sansoni, G., Trebeschi, M., \& Docchio, F. (2009). State-of-theart and applications of 3D imaging sensors in industry, cultural heritage, medicine, and criminal investigation. Sensors, 9(1), 568-601.

Scott, W., Roth, G., \& Rivest, J.-F. (2003). View planning for automated 3D object reconstruction inspection. ACM Computing Surveys, 35(1).

Srinivasan, V., Liu, H., \& Halioua, M. (1984). Automated phase-measuring profilometry of 3-D diffuse objects. Applied Optics, 23(18), 3105-3108.

Tang, S., \& Hung, Y. Y. (1990). Fast profilometer for the automatic measurement of 3-D object shapes. Applied Optics, 29(20), 3012-3018.

Volino, P., Thalmann, N. M., Jianhua, S., \& Thalmann, D. (1998). The Evolution of a 3D System for Simulating Deformable Clothes on Virtual Actors. MIRALab Copyright Information.

Zexiao, X., Jianguo, W., \& Qiumei, Z. (2005). Complete 3D measurement in reverse engineering using a multiprobe system. International Journal of Machine Tools and Manufacture, 45(12), 1474-1486.

Zhuang, H., Wang, K., \& Roth, Z. S. (1995). Simultaneous calibration of a robot and a hand-mounted camera. Robotics and Automation, IEEE Transactions on, 11(5), 649-660.

Webpages:

http://graphics.stanford.edu/projects/gantry

GOM ATOS Compact Scan 2Mlaser scanner:

http://www.gom.com/metrology-systems/system-

overview/atos-compact-scan.html 